\title{
EDITORIAL
}

\section{Antimicrobial Resistance: A Man Made Crisis}

\section{Background and Introduction}

Antibiotics and similar drugs, together called antimicrobial agents, have been used for the last 70 years, since first world war to treat patients who had infectious diseases. Since the 1940s, these drugs have greatly reduced illness and death from infectious diseases. Antibiotic use has been beneficial when prescribed and taken correctly. Their value in patient care is enormous. However, these drugs have been used so widely and for so long that the infectious organisms against which the antibiotics are designed to kill, have adapted to them, making the drugs less effective. Infections caused by resistant microorganisms often fail to respond to conventional treatment, resulting in prolonged illness and greater risk of death. About 440000 new cases of multidrug-resistant tuberculosis (MDR-TB) emerge annually, causing at least 150000 deaths. Resistance to earlier generation antimalarial medicines such as chloroquine and sulfadoxinepyrimethamine is widespread in most malaria-endemic countries. A high percentage of hospital-acquired infections are caused by highly resistant bacteria such as methicillin-resistant Staphylococcus aureus (MRSA). ${ }^{1}$ Inappropriate and irrational use of antimicrobial medicines provide favourable conditions for resistant microorganisms to emerge, spread and persist. Resistance to antimicrobials is a natural and inevitable biological phenomenon that can be amplified or accelerated by a variety of factors and practices that facilitate " selective pressure". The microbes which adapt and survive carry genes for resistance which can be passed on to the next generation of microbes and also in some bacteria, across different species. The selection pressure is utmost when antimicrobials are used irrationally in health and veterinary sectors. ${ }^{2,3,4}$

Antimicrobial resistance (AMR) reduces the effectiveness of treatment because patients remain infectious for longer, thus potentially spreading resistant microorganisms to others. Many infectious diseases risk becoming uncontrollable and could derail the progress made towards reaching the targets of the health-related United Nations Millennium Development Goals set for 2015. When infections become resistant to first-line medicines, more expensive therapies must be used. The longer duration of illness and treatment, often in hospitals, increases health-care costs and the financial burden to families and societies. The achievements of modern medicine are put at risk by AMR. Without effective antimicrobials for care and prevention of infections, the success of treatments such as organ transplantation, cancer chemotherapy and major surgery would be compromised. The growth of global trade and travel allows resistant microorganisms to be spread rapidly to distant countries and continents. ${ }^{5}$

\section{Global and Regional Status:}

About 440000 new cases of multidrug-resistant tuberculosis (MDR-TB) emerge annually, causing at least 150000 deaths. Extensively drug-resistant tuberculosis (XDR-TB) has been reported in 64 countries to date. ${ }^{6}$

Resistance to earlier generation antimalarial medicines such as chloroquine and sulfadoxine-pyrimethamine is widespread in most malaria-endemic countries. Falciparum malaria parasites resistant to artemisinins are emerging in South-East Asia; infections show delayed clearance after the start of treatment (indicating resistance) ${ }^{7}$

A high percentage of hospital-acquired infections are caused by highly resistant bacteria such as methicillinresistant Staphylococcus aureus (MRSA) and vancomycin-resistant enterococci. ${ }^{8}$

Resistance is an emerging concern for treatment of HIV infection, following the rapid expansion in access to antiretroviral medicines in recent years; national surveys are underway to detect and monitor resistance. ${ }^{9}$ 
Ciprofloxacin is the only antibiotic currently recommended by WHO for the management of bloody diarrhoea due to Shigella organisms, now that widespread resistance has developed to other previously effective antibiotics. But rapidly increasing prevalence of resistance to ciprofloxacin is reducing the options for safe and efficacious treatment of shigellosis, particularly for children. New antibiotics suitable for oral use are badly needed. ${ }^{10,11}$

AMR has become a serious problem for treatment of gonorrhoea (caused by Neisseria gonorrhoeae), involving even "last-line" oral cephalosporins, and is increasing in prevalence worldwide. Untreatable gonococcal infections would result in increased rates of illness and death, thus reversing the gains made in the control of this sexually transmitted infection. There has been a substantial change in the antimicrobial susceptibility of Neisseria gonorrhoeae. Thirty years back, gonorrhoea used to respond effectively to penicillin. Now the resistance to penicillin and fluoroquinolones is widespread across the Region. ${ }^{12}$

New resistance mechanisms, such as the beta-lactamase NDM-1, have emerged among several gram-negative bacilli. This can render powerful antibiotics, which are often the last defence against multi-resistant strains of bacteria, ineffective.

Resistance to first-line anti-TB drugs has become a concern for national TB control programmes.The population weighted mean of multi-drug resistant tuberculosis (MDR-TB) in the Region is 2.8 per cent (1.9-3.6\%) among new cases and 18.8 per cent (13.324.3\%) among previously treated cases. It is estimated that around 180, 000 cases of MDR-TB reside/occur annually in this Region with more than 80 per cent of these being in Bangladesh, India, Indonesia, Myanmar and Thailand.The drugs needed to treat MDR-TB are over 100 times more expensive than the first-line drugs used to treat non-resistant forms. In some countries, the high cost of such replacement drugs is prohibitive, with the result that some of the MDR-TB cases can no longer be treated. ${ }^{13}$

The generic antiretroviral (ART) drugs available in the Region are contributing greatly towards improving the survival rate of patients worldwide and in rendering HIV as a chronic but a manageable condition. Although the response to ART drugs is excellent when these are delivered at health facilities, there are reports of the emergence of resistance that is a serious cause of concern.

Resistant malaria has already become a major issue for a population of 400 million living in areas that expose them to a high risk of contracting it. Artemisinin-based combination therapies (ACT) have recently been introduced in virtually all countries in which malaria is endemic. However, surveillance data from the Thai Ministry of Public Health indicate that clinical failures of artemisinin-based therapies exist in the ThaiCambodian border, whereas efficacy with artesunatemefloquine along the western borders of Thailand remains high. ${ }^{14}$

Pentavalent antimonials (SbV) have been successfully used for treatment of kala-azar since the last six decades. Since the 1970s, however, their conventional dosages have failed to achieve the desired results with 60 per cent unresponsiveness being reported with the WHO regimen in Bihar (India). Pentamidine initially used as a second-line drug, acquired resistance (25\%) even with prolonged dosage. The newer oral drug, miltefosine is a potent antileishmanial drug with a longer half-life, a property likely to delay resistance. The evolution of resistance to this drug will cause havoc to the regional efforts to combat this disease. ${ }^{15}$

Cholera bacilli have acquired resistance to a number of antimicrobials. The resistance spectrum varies in different locales. In areas around New Delhi (India) extensive resistance to furazolidone, co-trimoxazole and nalidixic acid has been noted while tetracycline has remained effective. On the other hand, in Bangladesh, tetracycline resistance has been found to be frequent in prevalent Vibrio cholera. ${ }^{11}$

Streptococcus pneumoniae is the most common causative agent of pneumonias in children and adults in Asia. Till the1980s, almost all isolates of this organism used to be susceptible to penicillin. In 2006, in a hospital in Thailand, almost 69 per cent isolates of this bacterium were found to be penicillin resistant. ${ }^{16,17}$

Typhoid and paratyphoid fever continue to be important causes of illness and death, particularly among children and adolescents in the SEA Region where this disease is associated with poor sanitation and unsafe food and water. Shortly after the emergence of multidrug-resistant $S$. Typhi in this Region, case fatality rates approaching 
10 per cent (close to $12.8 \%$ recorded in pre-antibiotic era) were reported. ${ }^{18}$

More than 50 per cent isolates of Staphylococcus aureus in hospital settings are now methicillin resistant. In a study undertaken in a 1000 bedded hospital in Thailand, 48 per cent patients with bacteraemia due to resistant $S$. aureus died. Methicillin-resistant S. aureus (MRSA) is a major problem in hospital-associated infections in almost all countries in the SEA Region. ${ }^{19}$

Multiresistant klebsiellae, Pseudomonas and Acinetobacter species have given new dimensions to the problem of hospital-associated infections. A. baumannii has become an important pathogen in intensive care units. In a study done in Thailand, mortality in admitted patients due to imipenem-resistant A. baumannii was 52 per cent as compared to 19 per cent in those who were infected with the sensitive variant. Presence of a drug resistant gene $b l a_{\mathrm{NDM}-1}$ in several members of the family Enterbacteriaceae has given rise to organisms that are resistant to a large number of commonly used antimicrobial agents.

Several salmonellae were isolated from chicken carcasses imported into Bhutan, 40 of 42 Salmonella enteritidis exhibited resistance to more than 2 drugs. From clinically healthy cows in Thailand, 68 per cent of isolates of $S$. enterica were resistant to at least one antimicrobial and 6 per cent were multiresistant. A spread of multiresistant $S$. schwarzengrund from chickens to humans in Thailand and from imported Thai food products to persons in Denmark and the United States has been well documented. ${ }^{17,18}$

\section{Causes of AMR}

Inappropriate and irrational use of medicines provides favourable conditions for resistant microorganisms to emerge and spread. For example, when patients do not take the full course of a prescribed antimicrobial or when poor quality antimicrobials are used, resistant microorganisms can emerge and spread. ${ }^{19,} 20,21$

Underlying factors that drive AMR include:

- inadequate national commitment to a comprehensive and coordinated response, ill-defined accountability and insufficient engagement of communities;

- weak or absent surveillance and monitoring systems;

- inadequate systems to ensure quality and uninterrupted supply of medicines
- inappropriate and irrational use of medicines, including in animal husbandry:

- poor infection prevention and control practices;

- depleted arsenals of diagnostics, medicines and vaccines as well as insufficient research and development on new products.

\section{Policy to combat AMR:}

The emergence of AMR is a complex problem driven by many interconnected factors; single, isolated interventions have little impact. A global and national multi-sectoral response is urgently needed to combat the growing threat of AMR. ${ }^{21,22}$

WHO is engaged in guiding the response to AMR through:

- policy guidance, support for surveillance, technical assistance, knowledge generation and partnerships, including through disease prevention and control programmes;

- essential medicines quality, supply and rational use;

- infection prevention and control;

- patient safety;

- laboratory quality assurance.

WHO has selected combating antimicrobial resistance as the theme for World Health Day 2011. On this day, WHO issues an international call for concerted action to halt the spread of antimicrobial resistance and recommends a six-point policy package for governments.

WHO calls on all key stakeholders, including policymakers and planners, the public and patients, practitioners and prescribers, pharmacists and dispensers, and the pharmaceutical industry, to act and take responsibility for combating antimicrobial .

\section{Partners in AMR activities}

Some organizations are engaged in several areas of prevention and containment of antimicrobial resistance. A synergy between these can yield better results.

Some of the active organizations with their networks spread over several countries are:

International Network on Rational Use of Drugs (INRUD)

ReAct

INDEPTH 
Alliance for Prudent Use of Antibiotics (APUA)

Health Action International (Asia Pacific): HAIAP

GARP

The Member States in SEAR where some of these networks are active include Bangladesh, India, Indonesia, Maldives, Nepal and Thailand.

\section{Research needs and perspectives for Antimicrobial Resistance}

The issue of antimicrobial resistance requires research in following categories

- Basic research to bridge knowledge gap.

- Clinical/translational research to put new products into the health system.

- Operational/health systems research to convert information/knowledge into action.

Basic research in the following areas will benefit global efforts against antimicrobial resistance:

- Deciphering microbial genomics.

- How do the microbes cause disease?

- Ascertain dynamics of spread.

- Mechanism of antimicrobial resistance.

- Impact of agricultural/veterinary use.

- Discovery of new drug targets.

- Develop better diagnostics (viral vs bacterial).

- New vaccines.

Operational research on the following subjects is needed:

- Monitoring methodologies for antimicrobial resistance.

- Collect systematic data on compliance and proper public use.

- Understand impact of resistance on illness and economy.

- Determine factors that influence prescription habits.

- Elucidate behavioral aspects about self-medication and adherence and develop interventions to bring about change towards rational use.

The WHO Regional Strategy on Prevention and Containment of Antimicrobial Resistance and the initiative taken by the WHO Regional Office for SouthEast Asia. The following recommendations were made:

\section{For the World Health Organization, WHO should:}

1. Undertake advocacy with national authorities to establish national alliances against antimicrobial resistance;

2. Develop and disseminate generic protocols to facilitate generation of comparable epidemiological data on antimicrobial resistance and utilization of antimicrobials;

3. Facilitate cooperation between various players (government agencies, professionals, academia, NGOs, INGOs etc.) to enhance synergy between their actions and to obviate duplication of efforts;

4. Develop generic IEC material to create awareness amongst communities and obtain their active participation in the fight against AMR;

5. Through its WHO Collaborating Centre on AMR, collate and share global data and regional experiences on all aspects of antimicrobial resistance; The WHO CC should be supported to act as a Regional Clearing Centre and to coordinate multicentric studies in the Region.

6. Document and disseminate experiences gained within the Region and lessons learnt in combating AMR;

7. Support operational research on various aspects of antimicrobial resistance; and

8. Organize regional meetings on a regular basis for exchange of experiences within the Region.

For Member States in the South-East Asia Region Member States should:

1. Establish a national alliance against antimicrobial resistance with all key stakeholders as its members. The implementation of national efforts to prevent and contain antimicrobial resistance should be through a multisectorial national steering committee headed by the senior-most health executive and facilitated through advisory/ expert groups.

2. Designate a national focal point for antimicrobial resistance in the Ministry of Health.

3. Institute appropriate surveillance mechanisms in the health and veterinary sectors to generate reliable and actionable epidemiological information including baseline data and trends on antimicrobial resistance, utilization of antimicrobial agents and 
impact on the economy and health through designated national and regional reference centers

4. Discourage non-therapeutic use of antimicrobial agents in veterinary, agriculture and fishery practices as growth-promoting agents.

5. Develop national standard treatment and infection control guidelines and ensure their application at all levels of health care and veterinary services through training, continuous educational activities and establishment of functional drugs and therapeutic committees and hospital infection control committees in health facilities (with the focus on proven, cost-effective interventions such as isolation, hand washing etc.).

6. Undertake operational research for better understanding of the technical and behavioral aspects of prevention and control of antimicrobial resistance and utilize the outcomes of these research studies/interventions in policy and programmer development/ improvement in the national context.

7. Launch educational and awareness programmers for communities and different categories of health care professionals.

8. Strengthen communicable diseases control programmers to reduce disease burden and accord priority to the discipline of infectious diseases in medical education and health services.

\section{Recommendations and conclusions}

Several issues hamper the prevention and containment of antimicrobial resistance and the efficacy of these drugs to maintain their "wonder" status.

Some of these are neglected problem with a profound impact on health and economy; inadequate visibility at decision-making level, lack of education amongst prescribers and users, weak collaboration between stakeholders; poor or no systematic surveillance of resistance and consumption of antimicrobial agents', ineffective regulatory mechanism; lack of economic potential for pharmaceuticals to invest in development of new drugs; and abysmal infection control practices. ${ }^{4}$

(J Bangladesh Coll Phys Surg 2011; 29: 120-125)

\author{
Professor Quazi Tarikul Islam \\ FCPS, FACP, FRCP \\ Department of Medicine, Dhaka Medical College \\ Email:prof.tarik@gmail.com
}

\section{References:}

1. World Health Organization. Antimicrobialresistance 2010. http://www.who.int/mediacentre/factsheets/fs338/en/ index.html, accessed on August 21.

2. Arias CA, Murray BE. Antibiotic resistant bugs in the $21^{\text {st }}$ century - A clinical super-challenge. $N$ Engl J Med. 2009;360:439-42.

3. WHO Global Strategy for Containment of Antimicrobial Resistance. Geneva: WHO; 2001. World Health Organization. Available at: http://www.who.int/entity/csr/resources/ publications/drugresist/en/EGlobal_ Strat.pdf, accessed on May 5, 2010.

4. Prevention and containment of Antimicrobial resistance, Report of Regional Meeting ,Chiang Mai, Thailand June, 2010

5 World Health Organization Medicines: rational use of medicines. 2010. Available at: http://www.who.int/ mediacentre/factsheets/fs338/en/index.html, accessed on June 7, 2010.

6. MacPherson DW, Gushulak BD, Baine WB, Bala S, Gubbins PO, Holtom P, et al. Population mobility, globalization and antimicrobial drug resistance. Emerg Infect Dis. 2009;15:1727-32.

7. Vijaykadga S, Rojanawatsirivej C, Cholpol S, Phoungmanee D, Nakavej A, Wongsrichanalai C. In vivo sensitivity monitoring of mefloquine monotherapy and artesunatemefloquine combinations for the treatment of uncomplicated P.falciparum malaria in Thailand in 2003. Trop Med Int Health. 2006;11:211-9.

8. Ellerbroek L, Narapati D, Phu Tai N, Poosaran N, Pinthong $\mathrm{R}$, Sirimalaisuwan A, et al. Antibiotic resistance in salmonella isolates from imported chicken carcasses in Bhutan and from pig carcasses in Viet Nam. J Food Prot. 2010;73:376-9.

9. Aarestrup FM, Hendriksen RS, Jana L, Katie G, Kathryn T. International spread of multidrug-resistant Salmonella schwarzengrund in food products. Emerg Infect Dis. 2007;13:726-31.

10. Sharma NC, Mandal PK, Rohini Dhillon R, Jain M. Changing profile of Vibrio cholerae O1, O139 in Delhi and periphery. Indian J Med Res. 2007;125:633-40

11. Glass RI, Huq I, Alim AR, Yunus M. Emergence of multiple antibiotic-resistant Vibrio cholera in Bangladesh. J Infect Dis. 1980;142:939-42

12. Sutrisna A, Soebjakto O, Wignall FS, Kaul S, Limnios EA, Ray S, et al. Increasing resistance to ciprofloxacin and other antibiotics in Neisseria gonorrhoeae from East Java and Papua, Indonesia, in 2004-implications for treatment. Int J STD AIDS. 2006;17:810-12.

13. Nair N, Wares F, Sahu S. Tuberculosis in the South-East Asia Region. Bull World Health Organ. 2010;88:164-5.

14. Sharma SK, Dhooria S, Prasad KJ, George N, Ranjan S, Gupta $\mathrm{D}$, et al. Outcomes of antiretroviral therapy in a northern 
Indian urban clinic. Bull World Health Organ. 2010; 88: 2226.

15. Jha TK. Drug unresponsiveness and combination therapy for kala-azar. Indian J Med Res. 2006;123:389-98.

16. Song JH, Oh WS, Kang CI, Chung DR, Peck KR, Ko KS, et al. Epidemiology and clinical outcomes of community acquired pneumonia in adult patients in Asian countries: a prospective study by the Asian network for surveillance of resistant pathogens. Int J Antimicrobial Agents. 2008; 31: 107-14.

17. Srifuengfung S, Tribuddharat C, Champreeda P, Daniels J, Chokephaibulkit K, Wongwan N, et al. Antimicrobial susceptibility of Streptococcus pneumoniae isolated from patients with respiratory tract infections in Thailand. Southeast Asian J Trop Med Public Health. 2008;39:461-6.
18. Gupta A. Multidrug-resistant typhoid fever in children: epidemiology and therapeutic approach. Pediatr Infect Dis J. 1994; 13: 134-40

19. Tyagi A, Kapil A, Singh P. Incidence of methicillin resistant Staphylococcus aureus (MRSA) in pus samples at a tertiary care hospital. J Indian Acad Clin Med. 2008;9:33-5.

20. Jamulitrat S, Arunpan P, Phainuphong P. Attributable mortality of imipenem-resistant nosocomial Acinetobacter baumannii blood stream infection. J Med Assoc Thailand. 2009; 92: 413-9.

21. So AD, Gupta N, Cars O. Tackling antibiotic resistance. BMJ. 2010; 340: 1091-2.

22. European Medicine Agency. European Centre for Disease Prevention and Control Joint Technical Report:the bacterial challenge- time to react. 2009. 